\title{
LEARNING OBJECTS INTEROPERABILITY: THE ARIADNE EXPERIENCE
}

\author{
Philippe Vidal ${ }^{1}$, Julien Broisin ${ }^{1}$, Erick Duval ${ }^{2}$, and Stefaan Ternier ${ }^{2}$ \\ ${ }^{1}$ Université Paul Sabatier, Institut de Recherche en Informatique de Toulouse, 118 route de \\ Narbonne, 31062 Toulouse Cedex 4, France; ${ }^{2}$ Katholieke Universiteit Leuven, \\ Celestijnenlaan 200A, B-3001 Leuven, Belgium
}

\begin{abstract}
Currently hundreds of academic or commercial platforms are present on the eLearning market. Most of these systems are closed and allow neither share nor re-use. In this article we focus on the problem of interoperability, based on standards (some of which are still under development), and propose a solution to allow a system to share and reuse learning objects from heterogeneous sources. These solutions are implemented in our European and National projects, respectively ARIADNE and e-Mi@ge.
\end{abstract}

Key words: e-learning; learning objects; standard; Learning Object Metadata (LOM); interoperability; Learning Object Repository (LOR); Web-Based Learning Environment (WebLE); portability.

\section{INTRODUCTION}

Without standards, it is nearly impossible for an organization to integrate quality products into its structure or courses (Standards and Learning Objects, 2001). E-learning standards aim to ensure :

- Repositories interoperability, in order to support federated searches and, more generally, an interoperable infrastructure of heterogeneous learning object services.

- Re-use of resources and tools so that e-learning systems are as open as possible and that data and contents are as portable as possible (Vidal et al., October 2002). Indeed, various e-learning systems should be able to exploit the documents stored in a particular repository. 
To this aim, metadata standardization enables us to locate and make use of the various educational resources of the Internet more effectively, since metadata facilitate learning objects re-use and portability (Simard, 2002). Today various metadata sets are being used, including the Dublin Core Metadata Set ${ }^{1}$, or the Resource Description Framework (RDF Recommendation, 1999), or the Learning Object Metadata (LOM) ${ }^{2}$ standard.

In this article, we study the openings proposed by an e-learning system, the ARIADNE environment, a European project which has led to a selfsupportive association. This system is compatible with the LOM standard, itself based on early ARIADNE results. We first initiate an interoperability solution between the ARIADNE Knowledge Pool System (KPS) and two other kinds of Learning Objects Repository (LOR), before turning to the portability of Learning Objects (LO) stored in an ARIADNE LOR.

\section{INTEROPERABILITY STUDY}

\subsection{The ARIADNE system}

The ARIADNE system focuses on share and reuse of learning hypermedia documents (Vidal et al., July 2002). The heart of the ARIADNE environment is composed of a Knowledge Pool System (KPS) (Duval et al., 2001), compatible with the LOM standard that ARIADNE actively cofounded.

Two APIs (Application Program Interface) (Ternier et al., 2003), Indexation Tool and Query Tool illustrated on Figure 1, interact with the KPS. In order to be independent from the internal structure of the KPS so as to allow interoperability with third tools or other LORs, these APIs are based on Web Services, and more specifically on both the communication network protocol SOAP (Simple Object Access Protocol) and the XML (extensible Markup Language) standard.

There are many e-learning systems calling all upon different methods of storage. We now study a solution of interoperability between the ARIADNE KPS and two other systems of learning objects repository: Peer To Peer and Federated Search. 


\subsection{LORs interoperability}

\subsubsection{Peer To Peer}

A P2P (Peer To Peer) (Nejdl et al., 2002) (Ternier et al., 2002) application has both the client characteristics and the server's; this property allows the re-use of the components described in the previous section. A new intermediate layer acts like a bridge between the indexing and query tools and the KPS (Figure 1) (Ternier et al., 2003).

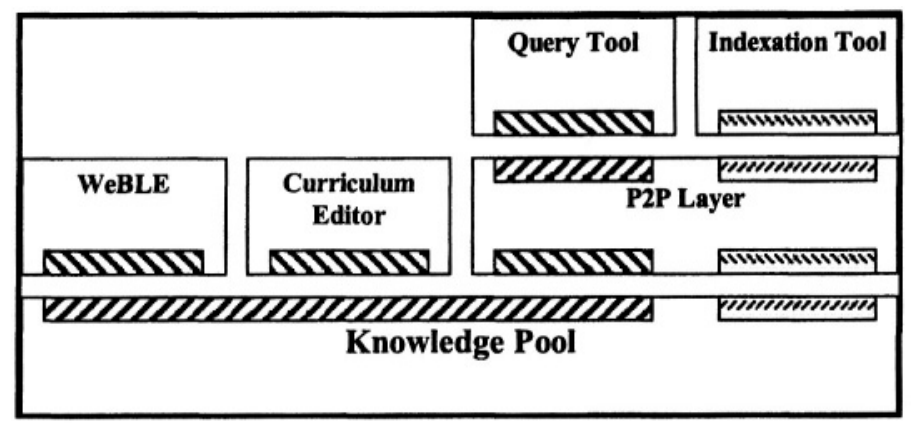

Figure 1. An intermediate P2P layer

When this layer receives a query, it locally carries out the query by using the KPS API, but also transfers the query to other actors. However, this mechanism is invisible for users; the use cases are unchanged. The local results as well as those coming from other actors are finally returned to the initiator of the query. Furthermore, the P2P layer takes care of the replication: when a user inserts a new LO from the indexing interface, the $\mathrm{P} 2 \mathrm{P}$ layer intercepts the request and chooses the node on which the LO will be stored.

\subsubsection{Federated Search}

As the P2P layer, the "Federated Search" layer can be used as an intermediate layer in our architecture (Figure 2). This layer accepts the queries coming from the query tool and transmits them to the KPS as well as to the other systems. If those other systems implement the ARIADNE interface, the transmission of the query is simple (other LOR1). Otherwise, an adapter must be provided in order to convert the ARIADNE API towards the protocol used by the other LOR (other LOR2). The queries for the LOs 
indexing towards this "Federated Search" system are transmitted to the KPS API.

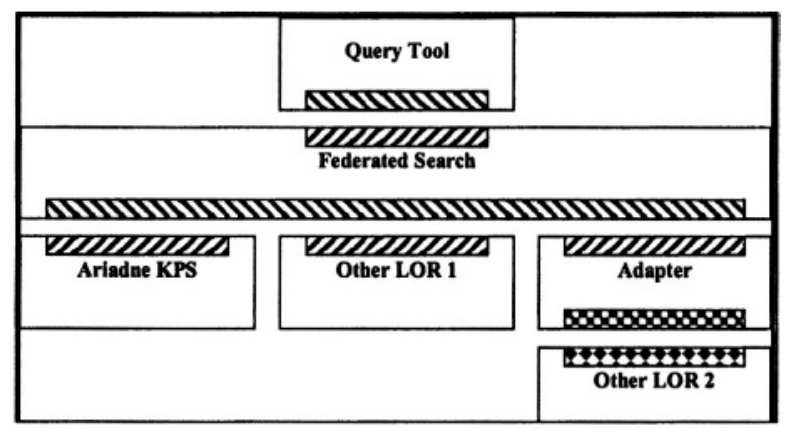

Figure 2. An intermediate Federated Search layer

After showing a possible solution to interoperability problems between heterogeneous LORs, we now discuss their interoperability with e-learning platforms.

\subsection{Interoperability between LOR and WebLE}

Once the resource has been described, it is inserted into the KPS and attributed a unique number (Alibert et al., 2003). The ARIADNE WeBLE (Web-Based Learning Environment), composed of a management interface and a learning interface, exploits these learning headers (Vidal et al., 2001).

In the ARIADNE environment, the system for storing LOs is independent from the e-learning platform (WebLE), which implies that any e-learning platform able to integrate LOM compatible resources can exploit all the documents contained in the ARIADNE KPS; such an example is proposed in the next section.

\section{AN EXAMPLE OF PORTABILITY : DEPLOYMENT OF LOS TOWARDS THE BLACKBOARD E-LEARNING PLATFORM}

The Blackboard ${ }^{3}$ platform is a learning management system able to integrate learning objects coming from other Learning Content Management Systems (LCMS). 
The Catholic University of Leuven has developed an extension which makes it possible for Blackboard to interact with the ARIADNE KPS (Vandepitte et al., 2003). This extension makes it possible to recopy resources stored in the ARIADNE KPS towards the Blackboard database, and to introduce some documents stored in the Blackboard system into the ARIADNE KPS. This application, or bridge, is a Web application made up of a reduced indexation tool.

\section{RELATED WORK}

We are currently developing a module which aims at allowing the communication between the ARIADNE KPS and the e-learning platform INES developed by the University of Picardy Jules Verne ${ }^{4}$. The extension's objective is to make it possible to download resources previously searched in the ARIADNE KPS and to introduce them into the INES database. The learning resources will then profit from the many functionalities this platform has. In addition, this module will propose the introduction of new learning objects into the ARIADNE KPS.

Accordingly, the "International e-Mi@ge"5 project, an e-learning training which delivers the MIAGE diploma, plans to adopt INES for the exploitation of the whole set of modules stored in the ARIADNE KPS.

\section{CONCLUSION AND PERSPECTIVES}

Thus, as we have seen throughout this article, we contributed to a solution in the field of pedagogical contents portability. Our work can be used to bring a response to these problems.

We are actively looking into solving the worrying problem of interoperability between the various platforms which affects all e-learning actors. Indeed, in addition to our participation in the LOM standardization, we are implied in the PROLEARN Network of Excellence financed by the Information Society Technology (IST) program of the European Commission, whose objective - among others - is to define an interconnection architecture for the various existing specifications.

Concerning our future work, we wish to improve communication between the ARIADNE knowledge pool system and the INES platform

4 http://www.u-picardie/fr/ cochard/e-miage

5 http://www.e-miage.org 
which provides a very complete student's learning follow-up. Our action should make it possible to solve the problem related to the learning followup of the student likely to sail on his own through identical learning environments.

\section{REFERENCES}

Alibert A., Vidal P., Marquié D., Baqué P., “Towards E-Leaming Platforms Interoperability : an Opening Solution", International Conference on Information Technology Based on Higher Education and Training, Marrakech, 2003 7-9 July, Marocco.

Duval E., Forte E., Cardinaels K., Verhoeven B., Durm V., Hrndrikx K., Forte M.W., Ebel N., Macowicz M., Warkentyne K., and Haenni F., "The ARIADNE Knowledge Pool System”, Communications of the A.C.M., vol. 44, $\mathrm{n}^{\circ}$ 5,2001 May, p.73-78.

Nejdl W., Wolf B., "EDUTELLA: A P2P Networking Infrastructure Based on RDF", Proceedings of WWW02 Conference, Hawai, 2002 7-11 May, USA.

Ternier S., Duval E., Vandepitte P., "LOMster: Peer-to-peer Learning Object Metadata", Proceedings of EdMedia-2002, 2002 June, p. 1942-1943.

Ternier S., Neuven F., Duval E., Macowicz M., Ebel N.: "Web Services for Learning Object Repositories : the case of ARIADNE Knowledge Pool System"., Proceedings of WWW03 Conference, Budapest, 2003 20-24 May, Hungary.

Resource Description Framework (RDF) Model and Syntax Specification, W3C recommendations, 1999, www.w3c.org/TR/REC-rdf-syntax.

Simard C.: "Normalisation de la formation en ligne : Enjeux, tendances et perspectives", Prepared for the «Agence Universitaire de la Francophonie » (AUF), Bureau Amérique du Nord, 2002 February.

Standards and Learning Objects, American Journal of Distance Education, vol. 15, $n^{\circ} 3,2001$.

Vandepitte P., Van Rentergem L., Duval E., Ternier S., Neven F, "Bridging an LCMS and an LMS: a Blackboard Building Block for the ARIADNE Knowledge Pool System", EdMedia 2003 - World Conference on Educational Multimedia, Hypermedia \& Telecommunications, 200327 June, Honolulu, Hawaii, USA.

Vidal P., Alibert A. : “ARIADNE: un réseau coopératif pour la conception auteur et 1 'apprentissage à distance", Institut de Recherche en Informatique de Toulouse, Toulouse, 2002 July.

Vidal P., Alibert A.: "Vers un enseignement à distance normalisé”, SITEF 2002 Espace Forum Village de le Recherche, Toulouse, 2002 October.

Vidal P., Apcher H., Marquié D.: "Intégration de services coopératifs dans un système d'enseignement à distance", Proceedings of the ARIADNE Foundation, vol. 1, n 1, 2001, p. 23-31. 